\title{
ERP Based Decision Fusion for AD Diagnosis Across Cohorts
}

\author{
Metin Ahiskali, Deborah Green, John Kounios, Christopher M. Clark and Robi Polikar*
}

\begin{abstract}
As the average life expectancy increases, particularly in developing countries, prevalence of neurodegenerative diseases has also increased. This trend is especially alarming for Alzheimer's disease (AD); as there is no cure to stop or reverse the effects of $\mathrm{AD}$. However, recent pharmacological advances can slow the progression of $A D$, but only if $A D$ is diagnosed at early stages. We have previously introduced an ensemble of classifiers based approach for combining event related potentials obtained from different electrode locations as an effective approach for early diagnosis of AD. We further expand this approach and analyze its robustness and stability in two ways: comparing the diagnostic accuracy on hand selected and cleaned data vs. standard automated preprocessing, but more importantly, comparing the diagnostic accuracy on two different cohorts, whose data are collected under different settings: a research university lab and a community clinic.
\end{abstract}

\section{INTRODUCTION}

$\mathrm{A}$ zheimer's disease is the fifth leading cause of death among the elderly, and the sixth leading cause of death among all age groups in the US. Given that there is no treatment available to stop or reverse the effects of $\mathrm{AD}$, and no definitive method for diagnosing the disease with certainty, combined with the availability of recently developed medications for slowing the progression of the disease, it has become increasingly important to diagnose AD at its earliest stages possible.

A neurodegenerative disease, $\mathrm{AD}$ is characterized by neuronal death, leading to declines in memory, motor skills and cognitive ability. While two misfolded proteins, $\beta$ amyloid and hyperphosphorylated $-\tau$, which cause plaques and neurofibrillary tangles, respectively, have been implicated as causing $\mathrm{AD}$, the genesis of these proteins is unknown. Perhaps more perplexing, recent studies have shown that removal of $\beta$-amyloid from the brain using targeted vaccination does not reverse the damage caused [1]. With overall life expectancy increasing, more notably in developed countries, the number of $\mathrm{AD}$ cases has grown tremendously since its first discovery over a century ago. The vast majority of $\mathrm{AD}$ patients are over the age of 65 , with an average $2 \%$ of cases affected under 65 (early onset AD), $19 \%$ between 75 and 84 , and $42 \% \sim 50 \%$ above the age of 85 . With over 5 million people suffering from $\mathrm{AD}$ alone, the enormous financial and emotional cost of the disease on the patient, family, and society in general has made $\mathrm{AD}$ a major health concern [2].

Currently, a definitive diagnosis of AD can only be made post-mortem by analyzing the brain tissue under the microscope for the presence of plagues and tangles, a method only available during an autopsy. More recently, cere-

This work is supported by NIH grants AG022272 and AG10124, PA Dept. of Health, SAP4100027296, and by NSF grant no ECS 0239090.

M. Ahiskali and R. Polikar are with Signal Processing and Pattern Recognition Laboratory, Dept. of Electrical and Computer Eng. at Rowan University, Glassboro, NJ 08028, USA. (e-mail: mahiskali@gmail.com).

C.M. Clark is with the Department of Neurology, University of Pennsylvania, Philadelphia, PA (e-mail: chris.clark@uphs.upenn.edu).

D. Green and J. Kounios are with the Dept. of Psychology, Drexel University, Philadelphia, PA(e-mail: dgreen@drexel.edu; Kounios@drexel.edu)

*Contact author: Robi Polikar, phone: (856) 256 - 5372; fax: (856) 2565241. E-mail: polikar@rowan.edu. brospinal fluid (CSF) concentrations of hyperphosphorylated- $\tau$ and $\beta$-amyloid proteins have been shown to be diagnostically informative biomarkers. However, such an analysis requires invasive, expensive, extremely painful and hence undesirable - lumbar puncture, which also requires specialty clinics, research or university hospitals.

The most common method of pre-mortem diagnosis is a longitudinal clinical evaluation, performed over a year or longer, which includes several batteries of memory tests as well as interviews of the patient and their caretakers. The primary path of diagnosis using this approach is through documenting cognitive decline over time and eliminating all other possible disorders. Such an evaluation is therefore subjective, tedious, requires expert neuropsychologists, and due to large amount of time involved in the process, is extremely expensive. While such an evaluation can achieve over $90 \%$ accuracy in $\mathrm{AD}$ diagnosis, when conducted by expert neurologists at large university hospitals or dementia clinics, geographical and financial restrictions generally limit most patients to smaller hospitals and clinics, where the diagnostic accuracies are estimated to be around $75 \%$, even with the benefit of longitudinal evaluations [3].

Given the prevalence of the disease, the expense and expertise needed for the less-than-perfect diagnosis, coupled with the availability of new drugs that work best if administered early, there is a compelling case for the urgent need for a reliable, inexpensive, non-invasive diagnostic tool that can be made available to community clinics, where most people seek medical help. The event related potentials (ERPs) obtained from the electroencephalogram (EEG) may provide just such a tool. Long overlooked due to its poor spatial resolution and difficulty of interpretation, recent advances in signal processing and machine learning have shown that early dismissal of ERPs as a potential biomarker were unfounded. In fact, several recent studies using the well-known oddball paradigm have demonstrated that the decreased amplitudes and increased latencies of the P300 component of ERPs - a positive peak that occurs around $300 \mathrm{~ms}$ after a stimulus is observed by the subject is linked to cognitive decline [4-7]. Various signal processing approaches on the raw EEG or the $\mathrm{P}_{300}$ has been conducted since then, verifying the presence of a statistical correlation, that showed limited success in patient specific diagnosis [8-10]. In our early work, we have shown that discrete wavelet coefficients of the ERPs, and not that of just the $\mathrm{P}_{300}$ components, are quite beneficial in patient specific AD diagnosis, particularly when the ERPs in response to different types of stimuli are combined [11-14].

Previously, we introduced an ensemble of classifiers based decision fusion approach, showing the feasibility of combining ERPs acquired from different electrodes for automated early diagnosis $[13 ; 15]$. In this contribution, we extend our analysis to include the robustness of the approach with respect to manual hand cleaning vs. automated preprocessing, but more importantly with respect to different cohorts. To the best of our knowledge, this is the first study of its kind that compares the performance on different cohorts whose data are acquired at different centers. 


\section{EXPERIMENTAL SETUP}

\section{A. Patient Cohorts}

Two different cohorts were recruited, which we will refer to as Cohort A and Cohort B in the rest of this paper. Cohort A subjects were primarily recruited between 2003 and 2006, with particular emphasis on AD subjects being in the earliest stages of the disease. Cohort B subjects were recruited between 2006 and 2009, where AD group represented a wider spectrum of disease stages. While there are many indicators to determine the severity of the disease, one of the simpler and effective tests is the Mini Mental State Exam (MMSE). The MMSE is a standardized test performed to access the subject's attention, immediate and short-term memory, language, as well as their ability to follow various written and verbal communication. For healthy individuals over the age of 80 , the median score is a 25 out of possible 30 total points (for no cognitive decline); however, this score is strongly proportional to number of years of education. The median MMSE is 29 , for those with at least 9 years of schooling, and 22 for those with zero to four years of education. A score of 19 or lower is a clear indicator of cognitive impairments and hence $\mathrm{AD}$ pathology [16]. Cohort $\mathrm{A}$, had $34 \mathrm{AD}$ patients with $\mu_{\mathrm{AGE}(\mathrm{AD})}=74$, $\mu_{\mathrm{MMSE}(\mathrm{AD})}=25$, and 37 controls $(\mathrm{CN})$ with $\mu_{\mathrm{AGE}(\mathrm{CN})}=76$, and $\mu_{\mathrm{MMSE}(\mathrm{CN})}=29$, indicating that the AD patients were in their earliest stages of the disease. For Cohort B, we had $27 \mathrm{AD}$ patients with $\mu_{\mathrm{AGE}(\mathrm{AD})}=77, \mu_{\mathrm{MMSE}(\mathrm{AD})}=23$, and 26 control subjects with $\mu_{\mathrm{AGE}(\mathrm{CN})}=76$, and $\mu_{\mathrm{MMSE}(\mathrm{CN})}=29$.

Cohort B subjects continue to be recruited, and over 100 subjects have been recruited so far. However, unlike Cohort A, Cohort B data acquisition is done - not in a carefully controlled research laboratory setting, but rather in a setting that mimics a community clinic. Hence the data are substantially noisier than those for Cohort A. Therefore, for this specific test, we chose a small subset of these patients, whose data were manually and carefully analyzed and cleaned by a specially trained EEG technician. We compared the performance of the proposed system on the identical subjects whose data were manually cleaned by the technician vs. automatically cleaned by a preprocessing scheme. Furthermore, while the Cohort A EEG data were obtained using 19 electrodes, Cohort B data were acquired using only 16 electrodes (using a simpler amplifier, mimicking one that may be available to community clinics).

For both Cohort A and B, inclusion criteria for $\mathrm{AD}$ group was satisfying the NINCDS-ADRDA criteria [17] for probable $\mathrm{AD}$, which includes a battery of memory tests (including MMSE), interviews with the subject and their caregivers, clinical dementia rating score of 0.5 or higher for $\mathrm{AD}$ cohort and 0 for the normal cohort. All subjects were over 60 years old. Exclusion criteria for both groups were evidence of any other central nervous system damage or use of sedatives, anxiolytic or antidepressants within 48 hours of ERP acquisition.

\section{B. The Oddball Paradigm and ERP Acquisition}

The ERPs acquired from the patients were collected using an auditory oddball paradigm protocol. Electrodes were placed according to 10-20 standard (Fig 1), whose impedances were kept below $20 \Omega$. Each subject was tested for 30 minutes with approximately three minutes of rest for every five minutes of testing. A $1 \mathrm{kHz}$ tone was presented to both ears, $60 \mathrm{~dB}$ above each subjects hearing threshold.

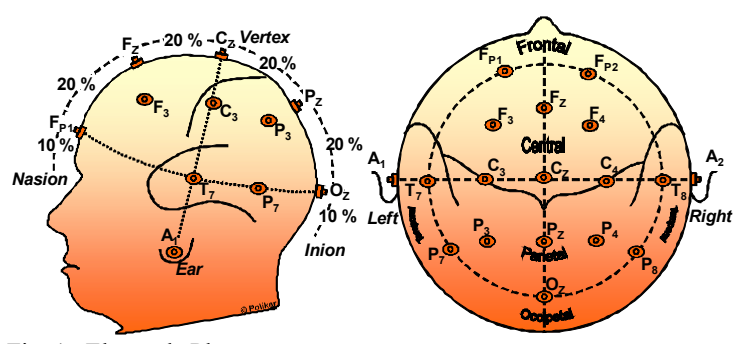

Fig. 1 -Electrode Placement

A total of 1,000 random stimuli were presented, $65 \%$ consisting of standard (non-target) tones of $1 \mathrm{kHz}, 20 \%$ as target tones of $2 \mathrm{kHz}$, and $15 \%$ as novel sounds. A random inter-stimulus interval ranging from 1.0 to 1.3 seconds was inserted. Standard and target stimuli were presented in $100 \mathrm{~ms}$ busts with a $5 \mathrm{~ms}$ on/offset envelope. The novel stimuli were environmental sounds about $200 \mathrm{~ms}$ long, each unique and never repeated. The subjects were instructed to press a button each time they heard the target tone only. The data was sampled at 256 samples /second.

\section{METHOD}

\section{A. Preprocessing \& Feature Extraction}

Cohort A data were hand processed by a trained technician, before the data were released to us for analysis - in the form of cleaned and averaged ERPs. For Cohort B data, we were given both the raw EEGs, as well as the manually cleaned and processed ERPs. Our simple automated preprocessing scheme consisted of the following: the data were first notch filtered at 59-61 Hz. Any recording with major artifacts were rejected using a $20^{\text {th }}$ order derivative based thresholding. ERPs were obtained from these EEG recordings, averaged and time-locked with $200 \mathrm{~ms}$ prestimulus and $800 \mathrm{~ms}$ post-stimulus intervals, to remove random variations in the signals. The pre-stimulus baseline was then removed from the entire ERP, with the final ERP of one second length of 256 samples per stimulus type, per electrode channel, per patient.

Since ERPs are nonstationary signals with time varying spectral components, we use the discrete wavelet transform coefficients for feature extraction, where ERPs were broken down into the following frequency bands:
$\boldsymbol{d}_{1}: 64 \sim 128 \mathrm{~Hz}(N=132)$
$\boldsymbol{d}_{5}: 4 \sim 8 \mathrm{~Hz}(N=14)$
$\boldsymbol{d}_{2}: 32 \sim 64 \mathrm{~Hz}(N=69)$
$\boldsymbol{d}_{6}: 2 \sim 4 \mathrm{~Hz}(N=10)$
$\boldsymbol{d}_{3}: 16 \sim 32 \mathrm{~Hz}(N=38)$
$\boldsymbol{d}_{7}: 1 \sim 2 \mathrm{~Hz}(N=8)$
$\boldsymbol{d}_{4}: 8 \sim 16 \mathrm{~Hz}(\mathrm{~N}=22)$
$\boldsymbol{a}_{7}: 0 \sim 1 \mathrm{~Hz}(N=8)$.

In this study, the decomposition levels 5-7 and the approximation level 7 were of primary focus. These bands were chosen since the ERP signals generally reside within the $(0-8 \mathrm{~Hz})$ frequency range, giving us four frequency bands to analyze for each novel and target tone responses.

\section{B. Ensemble System Definition}

An ensemble of classifiers based decision fusion approach was implemented, which consists of a group of classifiers trained on different feature spaces to create varying decision boundaries. Classifiers with varying errors can then be combined to reduce the overall system error [18]. We can consider such a system analogous to our natural decision making process to see multiple opinions before making a final decision; akin to seeking agreement between experts (who may have access to different sets of information) to obtain greater confidence in the final decisions. 
Decision fusion based data fusion refers to combination of decisions made by individual classifiers, as opposed to feature based data fusion where individual features are usually concatenated. We use a modified (augmented) stacked generalization approach to obtain feature level decision fusion, followed by a combination of such decisions to create the final across-features decision fusion.

\section{Stacked Generalization}

The primary goal of stacked generalization is to confirm or correct what has been learned by a group of preliminary (Tier-1) classifiers with the use of a meta-classifier. Any instance in a certain region of the feature space (e.g., near the decision boundary), may be more likely to be misclassified by certain classifiers than others. This trend can be learned by mapping the outputs of an ensemble of classifiers to their true labels (Fig 2.), thereby allowing the system to adapt to classifiers with varying performances.

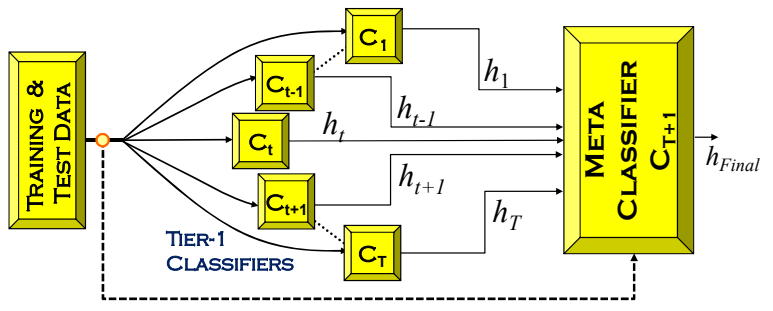

Fig. 2 - Stacked Generalization block diagram

\section{Augmented Stacked Generalization}

In modifying the basic stacked generalization algorithm, we augment the outputs of the Tier- 1 classifiers by concatenating the original data before training the metaclassifier (dashed line in Fig. 2), which we refer to as augmented stacked generalization (ASG). This process enriches the intermediate feature space used by the metaclassifier, which in turn aids in overall system performance. We implement a two stage training process, a leave-one-out (LOO) nested into a 5-fold cross validation. For each fold held out as test data, the remaining 4 folds are used in a LOO fashion to train the classifiers: $N-1$ instances are used to train the Tier-1 classifiers and the outputs of the Tier 1 classifiers on the remaining $N^{\text {th }}$ instance is used for training the meta-classifier. The system is then tested on the test block of the 5-fold cross validation. For each feature set (ERPs from different electrode locations, frequency bands and stimulus types), the entire process is then repeated 10 times, creating a different partition of 5 folds. Upon completion, we obtain a different ASG based system trained on different specific feature set (e.g. $\mathrm{NP}_{\mathrm{Z}} 12-$ Novel stimuli, $\mathrm{P}_{\mathrm{Z}}$ electrode, 1-2 Hz frequency band). This constitutes one decision fusion based expert for its respective feature set. Such experts are then combined using sum, and simple majority voting to obtain final decision fusion classification, combining different feature sets. Figure 3 illustrates the overall system diagram.

Multilayer perceptron based classifiers were used throughout the entire system, since these classifiers naturally provide an output that can be interpreted as a support for each class. Nine MLPs with 10 hidden layer nodes were used in Tier 1, and one MLP with 30 hidden layer nodes was used as the meta-classifier. All MLPs were trained with conjugate gradient based backpropagation, with a momentum term of 0.95 and an error goal of 0.05 .

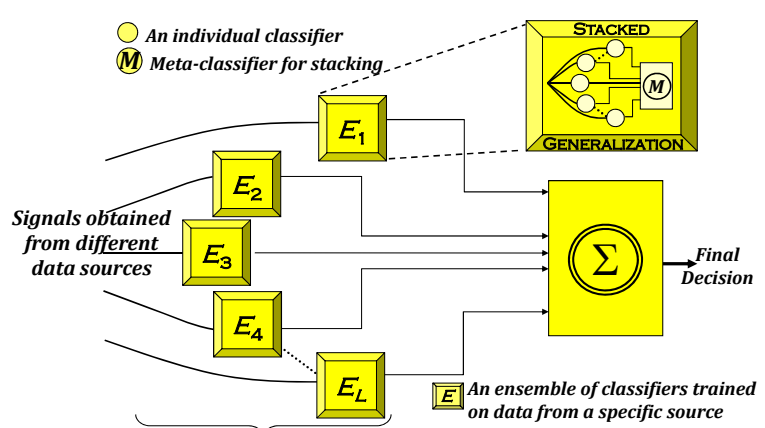

Feature - specific expert ensembles of classifiers (2) Classifier combination rule trained using stacked generalization

Fig. 3 - Overall system block diagram

\section{RESUlTs}

Since Cohort A and B utilize different electrodes, not all feature sets can be compared directly between the two. However, viable substitutions were made (see Tables 1 and 3 for feature set lists for each cohort), and the identical training and testing procedure was used for both.

Recall that Cohort B data underwent two different methods for cleaning and artifact rejection. First, it was hand cleaned by a trained EEG technician, manually removing or compensating for each artifact (e.g., muscular movement, eye blinks, etc.). In the second method, we employed a simple automated artifact rejection using derivative based thresholding and baselining. The analysis was then repeated on both sets of data to determine the robustness of the decision fusion based analysis with respect to different artifact rejection methods. This is because; the proposed automated system is intended for community clinics. Therefore, two performance metrics are given for all Cohort B calculations, each corresponding to either hand cleaning of the data or automated artifact rejection.

\section{A. Cohort A Diagnostic Accuracies}

The diagnostic accuracies for the highest performing 16 individual feature sets for Cohort A are shown in Table 1. The averages and $95 \%$ confidence intervals are based on 10 independent trials of 5-fold cross validation.

TABle 1 - Cohort A: Individual Feature Set PeRformances

\begin{tabular}{|c|c|c|c|c|c|}
\hline FS & Avg (\%) & $\begin{array}{l}\text { Best } \\
\text { Trial }\end{array}$ & FS & $\begin{array}{l}\text { Avg } \\
(\%)\end{array}$ & $\begin{array}{c}\text { Best } \\
\text { Trial }\end{array}$ \\
\hline $\mathrm{NP}_{\mathrm{Z}} 12$ & $70.1 \pm 1.2$ & 88.6 & $\mathrm{NT}_{8} 12$ & $64.0 \pm 1.6$ & 79.1 \\
\hline $\mathrm{NP}_{\mathrm{Z}} \mathbf{0 1}$ & $69.4 \pm 1.8$ & 77.1 & $\mathrm{TF}_{\mathrm{P} 2} 12$ & $63.0 \pm 1.2$ & 73.7 \\
\hline $\mathrm{NC}_{\mathrm{Z}} 12$ & $69.1 \pm 1.4$ & 78.0 & $\mathrm{NP}_{\mathrm{Z}} 24$ & $62.6 \pm 1.8$ & 79.7 \\
\hline $\mathrm{TP}_{\mathrm{Z}} 12$ & $67.5 \pm 1.6$ & 81.0 & $\mathrm{TP}_{3} 24$ & $62.5 \pm 1.0$ & 81.3 \\
\hline $\mathrm{TP}_{\mathrm{Z}} 24$ & $66.9 \pm 0.9$ & 89.9 & $\mathrm{TP}_{\mathrm{Z}} \mathbf{0 1}$ & $62.5 \pm 1.9$ & 79.7 \\
\hline $\mathrm{TF}_{8} 12$ & $66.5 \pm 1.9$ & 82.5 & $\mathbf{T P}_{3} 12$ & $62.4 \pm 1.2$ & 79.5 \\
\hline $\mathrm{NF}_{\mathrm{Z}} \mathbf{2 4}$ & $66.2 \pm 1.7$ & 75.1 & $\mathrm{TC}_{\mathrm{Z}} 24$ & $61.8 \pm 1.7$ & 79.4 \\
\hline $\mathrm{NC}_{\mathrm{Z}} 24$ & $64.7 \pm 1.2$ & 88.2 & $\mathrm{NO}_{\mathrm{z}} 12$ & $64.0 \pm 1.6$ & 79.1 \\
\hline
\end{tabular}

The better performing individual feature sets (FS) were then combined to complete the decision fusion based ensemble system. We chose three such combinations:

FS1: $\mathrm{NC}_{\mathrm{Z}} 12+\mathrm{NC}_{\mathrm{Z}} 24+\mathrm{NP}_{\mathrm{Z}} 24+\mathrm{NT}_{8} 12+\mathrm{NP}_{\mathrm{Z}} 24+\mathrm{TFP}_{2} 12+$

$\mathrm{TP}_{\mathrm{Z}} 01+\mathrm{TF}_{8} 12+\mathrm{TP}_{3} 24$

FS2: $\mathrm{NP}_{\mathrm{Z}} 12+\mathrm{NP}_{\mathrm{Z}} 24+\mathrm{NC}_{\mathrm{Z}} 24+\mathrm{NT}_{8} 12+\mathrm{TF}_{\mathrm{P} 2} 12+\mathrm{NC}_{\mathrm{Z}} 12$

FS3: $\mathrm{TF}_{\mathrm{P} 2} 12+\mathrm{TF}_{8} 12+\mathrm{TP}_{\mathrm{Z}} 01+\mathrm{NC}_{\mathrm{Z}} 12+\mathrm{NP}_{\mathrm{Z}} 24+\mathrm{NT}_{8} 12+\mathrm{NP}_{\mathrm{Z}} 12$

The results of the combinations are shown in Table 2 for sum and simple majority voting (SMV) based combination rules, which shows that the sum rule in general performing better, with statistical significance. 
TABLE 2 - COHORT A: DECISION FUSION DIAGNOSTIC ACCURACIES

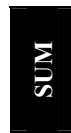

\begin{tabular}{|l|l|l|} 
& Avg (\%) & Best \\
\hline FS1 & $84.5 \pm 1.8$ & 94.7 \\
\hline FS2 & $81.3 \pm 1.7$ & 83.0 \\
\hline FS3 & $82.4 \pm 2.0$ & 87.0 \\
\hline
\end{tabular}

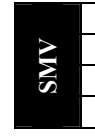

\begin{tabular}{|c|c|c|}
\hline & $\operatorname{Avg}(\%)$ & Best \\
\hline FS1 & $74.3 \pm 1.3$ & 82.1 \\
\hline FS2 & $71.8 \pm 2.1$ & 86.6 \\
\hline FS3 & $73.1 \pm 2.2$ & 82.0 \\
\hline
\end{tabular}

B. Cohort B Diagnostic Accuracies

Diagnostic accuracies for each feature set are shown in Table 3, for automated and hand cleaned preprocessing.

TABLE 3-COHORT B: INDIVIDUAL FEATURE SET PERFORMANCES

\begin{tabular}{|c|c|c|c|c|}
\hline \multirow{2}{*}{$\begin{array}{c}\text { Feature } \\
\text { Set }\end{array}$} & Avg (\%) & $\begin{array}{l}\text { Best } \\
\text { Trial }\end{array}$ & Avg (\%) & $\begin{array}{l}\text { Best } \\
\text { Trial }\end{array}$ \\
\hline & \multicolumn{2}{|c|}{ Computer } & \multicolumn{2}{|c|}{ Hand-Cleaned } \\
\hline NPZ12 & $62.9 \pm 1.5$ & 90.0 & $63.8 \pm 2.55$ & 90.0 \\
\hline NT812 & $65.8 \pm 2.4$ & 80.0 & $59.3 \pm 1.72$ & 80.0 \\
\hline NPZ24 & $59.3 \pm 0.9$ & 70.0 & $60.9 \pm 0.80$ & 76.9 \\
\hline NCZ24 & $62.7 \pm 1.2$ & 80.0 & $55.6 \pm 1.49$ & 80.0 \\
\hline NCZ12 & $65.6 \pm 2.5$ & 90.0 & $65.7 \pm 3.22$ & 80.0 \\
\hline NOZ12 & $58.8 \pm 2.3$ & 90.0 & $58.9 \pm 1.61$ & 80.0 \\
\hline TCZ24 & $60.5 \pm 2.0$ & 90.0 & $60.6 \pm 2.01$ & 90.0 \\
\hline TPZ12 & $57.8 \pm 1.1$ & 70.0 & $61.0 \pm 2.05$ & 90.0 \\
\hline TP312 & $57.5 \pm 2.3$ & 80.0 & $61.4 \pm 1.60$ & 80.0 \\
\hline NFZ24 & $60.9 \pm 2.4$ & 90.0 & $59.5 \pm 1.94$ & 80.0 \\
\hline TP324 & $65.8 \pm 2.2$ & 90.0 & $62.0 \pm 1.11$ & 90.0 \\
\hline TPZ24 & $65.8 \pm 1.3$ & 84.6 & $58.5 \pm 2.36$ & 80.0 \\
\hline NPZ01 & $59.5 \pm 1.4$ & 84.6 & $64.2 \pm 1.37$ & 80.0 \\
\hline TF312 & $58.7 \pm 1.6$ & 90.0 & $68.4 \pm 1.15$ & 80.0 \\
\hline TFP212 & $65.6 \pm 1.2$ & 90.0 & $63.6 \pm 2.34$ & 90.0 \\
\hline TPZ01 & $62.9 \pm 2.7$ & 100.0 & $57.5 \pm 0.82$ & 70.0 \\
\hline
\end{tabular}

Since different electrodes were used in Cohort B, the FS combinations vary slightly from those of Cohort A, with the only substitution being $\mathrm{TP}_{3} 12$ replacing $\mathrm{TF}_{8} 12$. Results of the final combinations, shown with each combination rule are shown in Table 4 for automated preprocessing, and in Table 5 for hand-cleaning based preprocessing.

TABLE 4-COHORT B: DECISION FUSION DIAGNOSTIC ACCURACIES BASED ON AUTOMATED PREPROCESSING

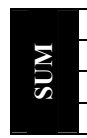

\begin{tabular}{|c|c|c|} 
& Avg (\%) & Best \\
\hline FS1 & $76.04 \pm 1.2$ & 81.12 \\
\hline FS2 & $79.11 \pm 1.8$ & 86.78 \\
\hline
\end{tabular}

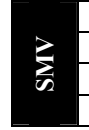

\begin{tabular}{|c|c|c|}
\hline & Avg (\%) & Best \\
\hline FS1 & $72.26 \pm 1.2$ & 82.83 \\
\hline FS2 & $71.88 \pm 1.9$ & 79.15 \\
\hline FS3 & $76.91 \pm 0.9$ & 86.14 \\
\hline
\end{tabular}

TABLE 5- COHORT B: DECISION FUSION DIAGNOSTIC ACCURACIESBASED ON HAND-CLEANED PREPROCESSING

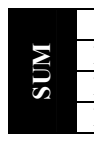

\begin{tabular}{|c|c|c|}
\hline & Avg (\%) & Best \\
\hline FS1 & $73.05 \pm 0.9$ & 80.00 \\
\hline FS2 & $78.16 \pm 1.9$ & 83.45 \\
\hline FS3 & $83.00 \pm 2.1$ & 94.99 \\
\hline
\end{tabular}

\begin{tabular}{|c|c|c|c|}
\hline \multirow{2}{*}{$\sum_{\text {U }}$} & & Avg (\%) & Best \\
\cline { 2 - 4 } & FS1 & $72.28 \pm 0.7$ & 79.91 \\
\cline { 2 - 4 } & FS2 & $73.52 \pm 1.2$ & 77.21 \\
\cline { 2 - 4 } & FS3 & $74.11 \pm 1.9$ & 76.55 \\
\hline
\end{tabular}

\section{Conclusions}

We make several observations from the results presented above. First, ASG based ensemble performance for any of the feature sets exceeds that of both community clinic diagnostic accuracy, as well as individual feature set performances (in Tables 1 and 3), demonstrating the feasibility of the approach. Second, the performance differences between Cohort A and Cohort B are not significant, nor are the differences between hand-cleaned preprocessing and automated preprocessing. This is important, indicating the ASG based decision fusion approach - when used with wavelet coefficients as features - is quite robust with respect to cohort differences as well preprocessing schemes. Third, the performance differences among the top three feature sets are all also very similar, with FS3 performing slightly better (with significance only in Cohort B), indicating that several different electrode combinations may be used for the analysis. The sum rule, however, performed significantly better than SMV across most comparisons.
Overall, we conclude that i) the approach is feasible as a first screening diagnosis tool to be used at community clinics, ii) there is in fact complementary information in ERPs obtained from different electrodes, which can be fused through decision fusion, and iii) the approach is quite robust to cohort and preprocessing variations. Future work will include expanding the analysis to a larger cohort, and training on one cohort and evaluating on the other.

\section{REFERENCES}

[1] S. Gilman, M. Koller, R. S. Black, and L. Jenkins, "Clinical effects of $\mathrm{A} \beta$ immunization (AN1792) in patients with $\mathrm{AD}$ in an interrupted trial," Neurology, vol. 64, pp. 1563-1572, 2005.

[2] Alzheimer's Assoc., 2009AD Facts and Figures, Available online: http://www.alz.org/ natinal/documents/report alzfactsfigures2009.pdf

[3] A. Lim, D. et al., "Clinico-neuropathological correlation of Alzheimer's disease in a community-based case series," J Am Geriatr Soc, vol. 47, no. 5, pp. 564-569, May1999.

[4] S. Yamaguchi, H. Tsuchiya, S. Yamagata, G. Toyoda, and S. Kobayashi, "Event-related brain potentials in response to novel sounds in dementia," Clinical Neurophys., vol. 111, no. 2, pp. 195-203, 2000.

[5] D. J. Linden, "The p300: where in the brain is it produced and what does it tell us?," Neuroscientist, vol. 11, no. 6, pp. 563-576, 2005.

[6] K. Bennys, F. Portet, J. Touchon, and G. Rondouin, "Diagnostic Value of Event-Related Evoked Potentials N200 and P300 Subcomponents in Early Diagnosis of Alzheimer's Disease and Mild Cognitive Impairment," J. Clinical Neurophys., vol. 24, no. 5, pp. 405-412, 2007.

[7] J. Polich, "Updating P300: An integrative theory of P3a and P3b," Clinical Neurophysiology, vol. 118, no. 10, pp. 2128-2148, Oct.2007.

[8] M. Lindau, V. Jelic, S. E. Johansson, C. Andersen, L. O. Wahlund, and O. Almkvist, "Quantitative EEG abnormalities and cognitive dysfunctions in frontotemporal dementia and Alzheimer's disease," Dement. Geriatr. Cogn Disord., vol. 15, no. 2, pp. 106-114, 2003.

[9] M. Karrasch, M. Laine, J. O. Rinne, P. Rapinoja, E. Sinerva, and C. M. Krause, "Brain oscillatory responses to an auditory-verbal working memory task in mild cognitive impairment and Alzheimer's disease," Int. Journal of Psychophysiology, vol. 59, no. 2, pp. 168-178,2006.

[10] T. Demiralp, A. Ademoglu, Y. Istefanopulos, C. Basar-Eroglu, and E. Basar, "Wavelet analysis of oddball P300," International Journal of Psychophysiology, vol. 39, no. 2-3, pp. 221-227, Jan.2001.

[11] G. Jacques, J. L. Frymiare, J. Kounios, C. Clark, and R. Polikar, "Multiresolution analysis for early diagnosis of Alzheimer's disease," IEEE Engineering in Medicine and Biology Conference (EMBC 2004), vol. 1, 2004, pp. 251-254.

[12] D. Parikh, N. Stepenosky, A. Topalis, D. Green, J. Kounios, C Clark, and R. Polikar, "Ensemble Based Data Fusion for Early Diagnosis of Alzheimer's Disease," 2005, pp. 2479-2482.

[13] R. Polikar, A. Topalis, D. Parikh, D. Green, J. Frymiare, J. Kounios, and C. M. Clark, "An ensemble based data fusion approach for early diagnosis of Alzheimer's disease," Information Fusion, vol. 9, no. 1, pp. 83-95, Jan.2008.

[14] R. Polikar, A. Topalis, D. Green, J. Kounios, and C. M. Clark, "Comparative multiresolution wavelet analysis of ERP spectral bands using an ensemble of classifiers approach for early diagnosis of Alzheimer's disease," Computers in Biology and Medicine, vol. 37, no. 4, pp. 542-558, Apr.2007.

[15] T. Patel, R. Polikar, C. Davatzikos, and C. M. Clark, "EEG and MRI data fusion for early diagnosis of Alzheimer's disease," Engineering in Medicine and Biology Society, 2008. EMBS 2008. 30th Annual International Conference of the IEEE, 2008, pp. 1757-1760.

[16] R. M. Crum, Anthony J.C., Bassett S.S., and M. Folstein, "Population-based norms for the Mini-Mental State Examination by age and educational level," Journal of American Medical Association, vol. 269, no. 18, pp. 2386-2391, 1993.

[17] G. McKhann, D. Drachman, M. Folstein, R. Katzman, D. Price, and E. M. Stadlan, "Clinical diagnosis of Alzheimer's disease: report of the NINCDS-ADRDA Work Group under the auspices of Department of Health and Human Services Task Force on Alzheimer's Disease," Neurology, vol. 34, no. 7, pp. 939-944, 1984.

[18] R. Polikar, "Bootstrap - Inspired Techniques in Computation Intelligence," Signal Processing Magazine, IEEE, vol. 24, no. 4, pp. 59-72, 2007. 